\title{
Minimal Quadratures for Functions of Low-Order Continuity
}

\author{
By L. W. Johnson and R. D. Riess
}

\begin{abstract}
An analog of Wilf's quadrature is developed for functions of low-order continuity. This analog is used to demonstrate that the order of convergence of Wilf's quadrature is at least $1 / n$.
\end{abstract}

1. Introduction. From the work done in minimal norm quadratures for Hilbert spaces of analytic functions by Wilf [7], Barnhill [1], Eckhardt [2], Richter [6], and others, it is natural to consider an extension of this concept for functions of low-order continuity. In this paper, we consider functions with a uniformly convergent FourierChebyshev expansion on the interval $[-1,1]$

$$
\begin{aligned}
f(x) & =\sum_{i=0}^{\infty} a_{i} T_{i}(x), \\
a_{i} & =\frac{2}{\pi} \int_{-1}^{1}\left(1-x^{2}\right)^{-1 / 2} f(x) T_{i}(x) d x,
\end{aligned}
$$

where $T_{i}(x)$ is the $i$ th degree Chebyshev polynomial of the first kind and the prime on the sum indicates the first term is to be halved. We also restrict $f(x)$ to have the property that $\sum_{\substack{i=0 \\ \infty}}^{\infty}\left|a_{i}\right|$ converges, e.g. when $f^{\prime}(x)$ is of bounded variation on $[-1,1]$. For error bounds of Gaussian quadrature for functions of this type, see Rabinowitz [5].

2. Minimal Quadratures. Let $\sum_{s-0}^{n} H_{s} f\left(x_{s}\right)$ be an $(n+1)$-point quadrature formula. We define $R_{n}(f)=\int_{-1}^{1} f(x) d x-\sum_{s=0}^{n} H_{s} f\left(x_{s}\right)$ and note from the expansion of $f(x)$ that $R_{n}(f)=\sum_{i=0}^{\infty} a_{k} R_{n}\left(T_{i}\right)$. Using both the triangle and Schwarz inequalities we obtain the error estimate

$$
\left|R_{n}(f)\right| \leqq\left(\sum_{i=0}^{k} \prime a_{i}^{2}\right)^{1 / 2}\left(\sum_{i=0}^{k} \prime \prime R_{n}\left(T_{i}\right)^{2}\right)^{1 / 2}+\sum_{i=k}^{\infty}\left|a_{i} R_{n}\left(T_{i}\right)\right|
$$

where the double prime indicates both first and last terms are to be halved.

If $f(x)$ satisfies mild smoothness restrictions (cf. Elliott, [3]), then the coefficients $a_{i}$ satisfy $\left|a_{i}\right| \leqq C / i^{2}$. In this case, since $R_{n}\left(T_{i}\right)$ is bounded for $i \geqq k$, the last term of the inequality is of order $1 / k$. Thus, it appears worthwhile to consider, as in Wilf [7], minimizing $W(n, k)$ where

$$
W(n, k)=\sum_{i=0}^{k}{ }^{\prime \prime} R_{n}\left(T_{i}\right)^{2}
$$

Received October 9, 1970, revised March 8, 1971.

AMS 1970 subject classifications. Primary 65D30.

Key words and phrases. Wilf's quadrature, optimal quadrature, order of convergence, low-order continuity.

Copyright @ 1971, American Mathematical Society 
We note $W(n, k)=0$ for $k<2 n+2$, since the problem is solved by Gauss-Legendre quadrature.

To minimize $W(n, k)$, of course, we must solve the $2 n+2$ simultaneous equations $\partial W(n, k) / \partial H_{s}=0$ and $\partial W(n, k) / \partial x_{s}=0,0 \leqq s \leqq n$. An analytic solution does not seem feasible, so we consider the less restrictive problem of choosing weights to minimize $W(n, k)$ with a given fixed set of nodes. In doing so we are able to answer a question posed by Wilf (see Section 4).

Solving $\partial W(n, k) / \partial H_{s}, 0 \leqq s \leqq n$, leads to the system

$$
\sum_{i=0}^{k} \prime \prime R_{n}\left(T_{i}\right) T_{i}\left(x_{s}\right)=0 ; \quad s=0, \cdots, n .
$$

Setting

$$
\begin{aligned}
\alpha_{i}=\int_{-1}^{1} T_{i}(x) d x & =-2 /\left(i^{2}-1\right), & & i \text { even, } \\
& =0, & & i \text { odd }
\end{aligned}
$$

thus (3) becomes

$$
\sum_{i=0}^{k} \prime \prime \alpha_{i} T_{i}\left(x_{s}\right)=\sum_{i=0}^{k} \prime \prime \sum_{i=0}^{n} H_{i} T_{i}\left(x_{i}\right) T_{i}\left(x_{s}\right) .
$$

If $H_{0}^{*}, \cdots, H_{n}^{*}$ satisfy (4), then $\sum_{s=0}^{n} H_{s}^{*} f\left(x_{s}\right)$ is called a minimal quadrature.

Let $g_{k}(x)=\sum_{i=0}^{\prime \prime k} \alpha_{i} T_{i}(x)$ and $f_{k}(x)=\sum_{i=0}^{\prime \prime k} R_{n}\left(T_{i}\right) T_{i}(x)$. Then,

$$
R_{n}\left(g_{k}\right)=\sum_{i=0}^{k} \prime \prime \alpha_{i} R_{n}\left(T_{i}\right)=\int_{-1}^{1} f_{k}(x) d x=R_{n}\left(f_{k}\right)-\sum_{s=0}^{n} H_{s} f_{k}\left(x_{s}\right) .
$$

If $H_{0}, \cdots, H_{n}$ is a solution of (4), then (3) the quadrature sum is zero. Further, as $R_{n}\left(f_{k}\right)=W(n, k)$, we have $R_{n}\left(g_{k}\right)=W(n, k)$ so $W(n, k)$, for any minimal quadrature, is the error made in approximating the integral of $g_{k}(x)$. We note here that $\sum_{i=0}^{\prime \infty} \alpha_{i} T_{i}(x)$ is the Fourier-Chebyshev expansion for $F(x)=\frac{1}{2} \pi\left(1-x^{2}\right)^{1 / 2}$ on $[-1,1]$, and since $F(x)$ is continuous and of bounded variation the series is uniformly convergent.

Let $H$ denote the $(n+1)$-dimensional vector $H=\left(H_{0}, \cdots, H_{n}\right)$ and define $\varphi: E^{n+1} \rightarrow E^{k+1}$ by $\varphi(H)=\left(R_{n}\left(T_{0}\right), \cdots, R_{n}\left(T_{k}\right)\right)$, where $R_{n}\left(T_{i}\right)=\alpha_{i}-\sum_{s=0}^{n} H_{s} T_{i}\left(x_{s}\right)$. It is immediate from Hilbert space properties that there is a unique point $H^{*}$ in $E^{n+1}$ such that $\left\|\varphi\left(H^{*}\right)\right\|_{2}$ is minimal. Thus, the existence of a unique minimal quadrature is guaranteed.

3. Special Case. When $k=n$, the minimal quadrature is of course the interpolatory quadrature on $x_{0}, \cdots, x_{n}$. In the case $x_{i}=\cos (i \pi / n)$, the interpolatory quadrature is Clenshaw-Curtis quadrature. If we use the well-known orthogonality properties for $T_{i}(x)$ in (4) with $x_{i}=\cos (i \pi / n)$, we obtain immediately $\frac{1}{2} n H_{i}=g_{n}\left(x_{i}\right)$, $i=1, \cdots, n-1$, and $n H_{i}=g_{n}\left(x_{i}\right)$ for $i=0$ or $n$. These are the same expressions found by Imhof [4], which he used to show the Clenshaw-Curtis weights were positive.

4. Improvement of a Result of Wilf. In [7] Wilf minimizes $W_{n}=\sum_{k=0}^{\infty} R_{n}\left(x^{k}\right)^{2}$. Let $R_{n}^{*}$ denote the remainder for optimal quadrature in the set of functions analytic in $|z|<1$ and $\mathscr{L}^{2}$ on the unit circle, and let 


$$
\|f\|^{2}=\frac{1}{2 \pi} \int_{0}^{2 \pi}\left|f\left(e^{i \theta}\right)\right|^{2} d \theta .
$$

Thus $\left|R_{n}^{*}(f)\right| \leqq W_{n}^{1 / 2}|| f||$. Wilf was unable to give explicit solutions for the weights and nodes, but was able to show that $W_{n}$ is the magnitude of the error in integrating $x^{-1} \log (1-x)^{-1}$ by the minimal formula. He derives the result $W_{n} \leqq O(\ln (n) / n)$ and leaves as an open question whether this result can be improved. On $[0,1]$ the ClenshawCurtis weights and nodes are, respectively,

$$
\begin{aligned}
& w_{i}=\frac{4}{n+1} g_{n+1}\left(x_{i}\right), i=1, \cdots, n, \\
& =\frac{2}{n+1} g_{n+1}\left(x_{i}\right), i=0 \text { or } n+1 ; x_{i}=\left[\cos \frac{i \pi}{2(n+1)}\right]^{2}, i=0, \cdots, n+1 .
\end{aligned}
$$

For ease of computation, and since $g_{n+1}(x)$ is uniformly convergent to $\frac{1}{2} \pi\left(1-x^{2}\right)^{1 / 2}$ on $[0,1]$, we shall use instead the weights

$$
\begin{aligned}
H_{i} & =\frac{2 \pi}{n+1}\left(1-\left(x_{i}\right)^{2}\right)^{1 / 2}, & i=1, \cdots, n, \\
& =\frac{\pi}{n+1}\left(1-\left(x_{i}\right)^{2}\right)^{1 / 2}, & i=0 \text { or } n+1,
\end{aligned}
$$

and we note $H_{0}=x_{n+1}=0$.

Since Clenshaw-Curtis quadrature is exact for polynomials of degree less than $n+2$,

$$
\sum_{s=0}^{n+1} w_{s}\left(x_{s}\right)^{k}=\frac{1}{k+1} \text { for } 0 \leqq k \leqq n+1
$$

Then

$$
\begin{aligned}
\sum_{k=0}^{n} R_{n}\left(x_{k}\right)^{2} & \equiv \sum_{k=0}^{n}\left(1 /(k+1)-\sum_{s=1}^{n} H_{s} x_{s}^{k}\right)^{2} \\
& =\sum_{k=0}^{n}\left(\sum_{s=0}^{n+1}\left(w_{s}-H_{s}\right) x_{s}^{k}\right)^{2} \\
& \leqq \sum_{k=0}^{n}\left(w_{0}+\sum_{s=1}^{n}\left|w_{s}-H_{s}\right|\right)^{2}
\end{aligned}
$$

For $1 \leqq s \leqq n$,

$$
\begin{aligned}
\left|H_{s}-w_{s}\right| & =(4 /(n+1))\left|\sum_{i=n+1}^{\infty} \alpha_{i} T_{i}\left(x_{s}\right)\right| \\
& \leqq(4 /(n+1)) \sum_{i=n+1}^{\infty}\left|\alpha_{i}\right| \leqq 4 /\left(n^{2}-1\right) .
\end{aligned}
$$

Since $w_{0}=1 /\left((n+1)^{2}-1\right)$, then by inserting these bounds we get

$$
\sum_{k=0}^{n} R_{n}\left(x^{k}\right)^{2} \leqq(n+1)(1+4 n)^{2} /\left(n^{2}-1\right)^{2} \leqq C_{1} / n .
$$


Now, for $k>n$, define

$$
Q_{n}\left(x^{k}\right) \equiv \sum_{s=1}^{n} H_{s} x_{s}^{k} \leqq \frac{2 \sqrt{ } 2 \pi}{n+1} \sum_{s=1}^{n} \sin \left(\frac{s \pi}{2 n+2}\right)\left(\cos \left(\frac{s \pi}{2 n+2}\right)\right)^{2 k} .
$$

In what follows we will show that $Q_{n}\left(x^{k}\right)=O(1 / k)$ for $k>n$. We first show that if $n$ is sufficiently large and $k \geqq(n+1)^{2}$, then $k Q_{n}\left(x^{k}\right)$ is bounded independently of $k$. We then show by an integral bound that $Q_{n}\left(x^{k}\right)=O(1 / k)$ for the remaining $k$ in $\left(n(n+1)^{2}\right)$.

First let us assume that $k \geqq(n+1)^{2}$, then

$$
y(k) \equiv k Q_{n}\left(x^{k}\right) \leqq \sum_{s=1}^{n} \sin \left(\frac{s \pi}{2 n+2}\right) V_{s}(k),
$$

where $V_{s}(k)=k(\cos (s \pi /(2 n+2)))^{2 k}$. Then it is easily verified that for $n$ sufficiently large (say $n \geqq M$ ) and $k \geqq(n+1)^{2}, V_{\bullet}^{\prime}(k)<0$, and thus $y(k)$ is bounded for all such $k$.

Now we consider a fixed $n \geqq M$ and any $k<(n+1)^{2}$. We define

$$
z(s)=\left(\frac{2 \sqrt{ } 2 \pi}{n+1}\right) \sin \left(\frac{s \pi}{2 n+2}\right)\left(\cos \left(\frac{s \pi}{2 n+2}\right)\right)^{2 k} .
$$

Then $z^{\prime}\left(s^{*}\right)=0$ for $\tan \left(s^{*} \pi /(2 n+2)\right)=(1 / 2 k)^{1 / 2}$, and $s^{*}$ is unique in $(0, n+1)$. Thus if $m$ is the greatest integer in $s^{*}$, and since $z(s) \geqq 0$ in $(0, n+1)$ and is maximal at $s^{*}$

$$
\sum_{s=1}^{m-1} z(s)+\sum_{s=m+2}^{n} z(s) \leqq \int_{0}^{n+1} z(t) d t<\frac{2 \sqrt{ } 2}{k}
$$

Since $z\left(s^{*}\right) \leqq(2 \sqrt{ } 2 \pi /(n+1))(2 k+1)^{-1 / 2}$, then

$$
Q_{n}\left(x^{k}\right) \leqq \sum_{s=1}^{n} z(s) \leqq \frac{2 \sqrt{ } 2}{k}+2 z\left(s^{*}\right)<\frac{2(\sqrt{ } 2+2 \pi)}{k}
$$

Thus, combining these two cases with $C_{2}=2(\sqrt{ } 2+2 \pi)$,

$$
\begin{aligned}
\sum_{k=n+1}^{\infty} R_{n}\left(x^{k}\right)^{2} & \leqq \sum_{k=n+1}^{\infty}\left(1 /(k+1)^{2}+2 C_{2} / k(k+1)+C_{2}^{2} / k^{2}\right) \\
& =O(1 / n) .
\end{aligned}
$$

Combining this with (5) we get the desired result,

$$
W_{n} \leqq \sum_{k=0}^{\infty} R_{n}\left(x^{k}\right)^{2}=O(1 / n)
$$

Reflection on the magnitude of $R_{n}^{*}\left(x^{k}\right)^{2}$, i.e. $\left(1 /(k+1)-Q_{n}^{*}\left(x^{k}\right)\right)^{2}$, and the number of free parameters available leads us to conjecture that $O(1 / n)$ is the best possible bound for $W_{n}$.

Department of Mathematics

Virginia Polytechnic Institute and State University

Blacksburg, Virginia 24061 
1. R. E. Barnhill, J. E. Dennis \& G. M. Nielson, "A new type of Chebyshev quadture," Math. Comp., v. 23, 1969, pp. 437-441. MR 39 \#3698.

2. U. ECKHARDT, "Einige Eigenschaften Wilfscher Quadraturformeln," Numer. Math., v. 12, 1968, pp. 1-7. MR 41 \#1217.

3. D. L. ElliotT, "A Chebyshev series method for the numerical solution of Fredholm integral equations," Comput J., v. 6, 1963/64, pp. 102-111. MR 27 \#5386.

4. J. P. IMHOF, "On the method for numerical integration of Clenshaw and Curtis," Numer. Math., v. 5, 1963, pp. 138-141. MR 28 \#15.

5. P. Rabinowitz, "Error bounds in Gaussian integration of functions of low-order continuity," Math Comp., v. 22, 1968, pp. 431-434. MR 37 \#2447.

6. N. RICHTER, "Properties of minimal integration rules," SIAM J. Numer. Anal., v. 7, 1970, pp. 67-79. MR 41 \#4804.

7. H. S. WILF, "Exactness conditions in numerical quadrature," Numer. Math., v. 6, 1964, pp. 315-319. MR 31 \#4178. 